\title{
Perfil socioeconômico dos portadores de glaucoma no serviço de oftalmologia do hospital universitário da Universidade Federal de Juiz de Fora - Minas Gerais - Brasil
}

\author{
Socioeconomic profile of individuals presenting with glaucoma in the service of \\ ophthalmology of the University Hospital of the Federal University of \\ Juiz de Fora - Minas Gerais - Brazil
}

Cristiana Moraes Ramalho'

Lúcio Nei Ribeiro ${ }^{2}$

Lucrecia Santos Olivieri ${ }^{3}$

Jaqueline Aparecida da Silva ${ }^{4}$

Thiago Cardoso Vale ${ }^{5}$

Wesley de Paula Duque ${ }^{6}$
Trabalho realizado no Serviço de Oftalmologia, Departamento de Medicina Interna da Universidade Federal de Juiz de Fora - Juiz de Fora - Minas Gerais (MG) - Brasil.

Professora da Disciplina de Oftalmologia da Universidade Federal de Juiz de Fora - UFJF - Juiz de Fora (MG) - Brasil. Doutoranda em Oftalmologia pela Universidade Federal de Minas Gerais - UFMG - Belo Horizonte (MG) - Brasil.

Assistente voluntário do Serviço de Oftalmologia do Hospital Universitário da UFJF - Juiz de Fora (MG) Brasil.

Estudante de Medicina da UFJF - Juiz de Fora (MG) Brasil.

Estudante de Medicina da UFJF - Juiz de Fora (MG) Brasil.

Estudante de Medicina da UFJF - Juiz de Fora (MG) Brasil.

Estudante de Medicina da UFJF - Juiz de Fora (MG) Brasil.

Endereço de correspondência: Wesley de Paula Duque. Rua Mariano Procópio, 1396 - Apto. 603 - Juiz de Fora (MG) - CEP 36080-010

E-mail: wesley@nextwave.com.br

Recebido para publicação em 05.09.2006

Última versão recebida em 17.05.2007

Aprovação em 22.06.2007

\begin{tabular}{|l|}
\hline RESUMO \\
\hline Objetivo: Avaliação do perfil socioeconômico dos pacientes porta- \\
dores de glaucoma primário em acompanhamento no serviço de oftal- \\
mologia do hospital universitário da Universidade Federal de Juiz de \\
Fora (MG), Brasil. Métodos: Aplicação de um questionário em 100 \\
pacientes durante suas consultas no serviço de oftalmologia do hospital \\
universitário da Universidade Federal de Juiz de Fora, no período de \\
abril de 2005 a junho de 2006. Resultados: Observou-se que $84 \%$ dos \\
pacientes eram pensionistas ou aposentados, $86 \%$ recebiam de 1 a 2 \\
salários mínimos, 29\% gastavam de 26 a 75 reais/mês com o tratamento \\
do glaucoma, 78\% apresentavam co-morbidades e $41 \%$ já haviam \\
suspendido o tratamento por falta de condições financeiras para a \\
compra das medicações. Conclusão: A população em estudo, de baixo \\
nível socioeconômico, apresenta dificuldades de adesão terapêutica \\
por, principalmente, problemas financeiros e presença de co-mor- \\
bidades. Os autores reforçam a urgente necessidade de adoção de \\
medidas sociopolíticas que facilitem o acesso à medicação antiglau- \\
comatosa, determinando maior aderência terapêutica.
\end{tabular}

Descritores: Glaucoma; Glaucoma de ângulo aberto; Hipertensão ocular; Glaucoma de ângulo fechado/epidemiologia

\section{INTRODUÇÃO}

O glaucoma é uma neuropatia insidiosa que cursa, na maioria das vezes, com aumento pressórico intra-ocular (valor normal: de 10 a $20 \mathrm{mmHg})^{(1)}$. Acarreta a morte de células ganglionares da retina com conseqüente dano ao nervo óptico e perda de campo visual, principalmente periférico. Embora raros, podem ocorrer casos de glaucoma com pressão intra-ocular (PIO) normal, que se devem a uma doença específica do nervo óptico ${ }^{(1-2)}$.

O glaucoma é um problema de saúde pública e uma das mais importantes causas de cegueira no Brasil e no mundo ${ }^{(1,3)}$. Segundo a Organização Mundial de Saúde (OMS), o glaucoma é a terceira maior causa de cegueira na população mundial, sendo responsável por 2,4 milhões de novos casos ao ano $^{(3-5)}$. Estima-se que 66,8 milhões de indivíduos no mundo são acometidos pelo glaucoma primário de ângulo aberto (GPAA), sua forma mais preva- 
810 Perfil socioeconômico dos portadores de glaucoma no serviço de oftalmologia do hospital universitário da Universidade Federal de Juiz de Fora - Minas Gerais - Brasil

lente, responsável por cegueira bilateral em $10 \%{ }^{(6)}$. Estima-se que 900 mil brasileiros, com idade acima de 40 anos, apresentem glaucoma e cerca de $20 \%$ dos indivíduos que descobrem a doença em campanhas, não retornam ao médico $^{(7)}$.

Autores de um estudo conduzido na Holanda enfatizaram que a educação de pacientes portadores de glaucoma com nível socioeconômico mais baixo deveria ser dirigida preferencialmente aos fatores de risco e conseqüências da doença $^{(8)}$. Os mesmos autores concluíram que o nível de conhecimento geral sobre o glaucoma e seu tratamento está diretamente relacionado a resultados positivos na evolução da doença. O grupo de baixo nível socioeconômico necessita de maior informação sobre a assistência e aspectos práticos do glaucoma, freqüentemente subestimado.

Diante desse contexto, o presente estudo avalia o perfil socioeconômico dos pacientes portadores de glaucoma primário em acompanhamento no serviço de oftalmologia do hospital universitário da Universidade Federal de Juiz de Fora (MG), Brasil.

\section{MÉTODOS}

Durante o período de abril de 2005 a junho de 2006, foi aplicado um questionário (Anexo 1) a 100 pacientes portadores de glaucoma assistidos no serviço de oftalmologia, no departamento de medicina interna do hospital universitário da Universidade Federal de Juiz de Fora. Os pacientes foram informados dos objetivos do questionário e alertados de que a continuação do seu acompanhamento não esteve condicionada à resposta ao questionário. O mesmo foi aplicado por acadêmicos de medicina, após a consulta oftalmológica no hospital supracitado.

Os critérios de inclusão foram: a) idade acima de 18 anos; b) acompanhamento ambulatorial na instituição independen-

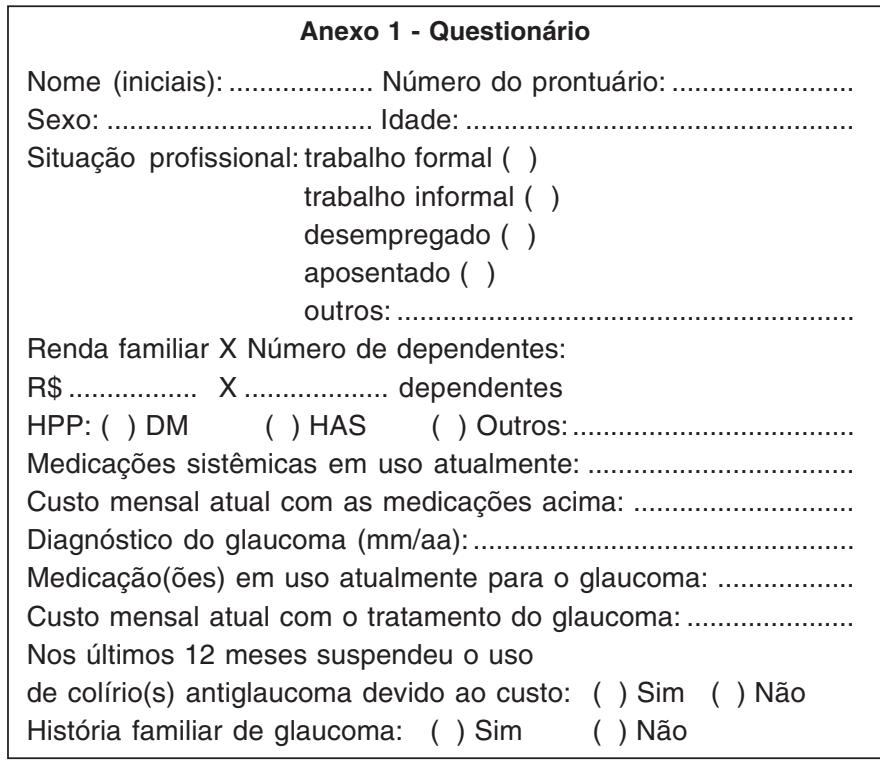

te da duração e estágio da doença; c) diagnóstico de GPAA e glaucoma primário de ângulo fechado (GPAF), independentes de outras enfermidades ametrópicas; d) uso de colírio antiglaucomatoso; e) despesa mensal com custo da medicação; f) concordância na participação do estudo por livre consentimento.

Os critérios de exclusão foram: a) a não concordância com a participação do estudo; b) recebimento de auxílio total, patrocínio ou doação da medicação antiglaucomatosa; c) não utilização de medicação antiglaucomatosa; d) diagnóstico de glaucoma congênito ou secundário.

Os dados foram avaliados pela freqüência estatística dentro da amostra e comparados aos da literatura. O estudo foi aprovado pelo comitê de ética da Universidade Federal de Juiz de Fora (848.154.2006).

\section{RESULTADOS}

Foram avaliados 100 pacientes portadores de glaucoma primário atendidos no serviço de oftalmologia do hospital universitário de Juiz de Fora no período de abril de 2005 a junho de 2006. A população apresentou uma média de idade de 61,3 anos +/- 13,6 anos (variando entre 18 e 91). Setenta por cento dos entrevistados eram do sexo feminino e $30 \%$, do masculino. Na distribuição entre o sexo e a idade dos entrevistados, obtivemos como resultado (Figura 1).

Trinta por cento dos pacientes apresentavam glaucoma com menos de 2 anos de evolução. Entre 2 e 5 anos, observouse $34 \%$ da amostra. Entre 6 e 10 anos de doença, 11\% dos casos. Acima de 10 anos, 25\% dos casos.

Oitenta e quatro por cento dos entrevistados eram pensionistas ou aposentados, enquanto que $10 \%$ e $6 \%$ tinham situação profissional formal (carteira assinada) e informal, respectivamente.

Quando questionados em relação à renda familiar e número de pessoas no domicílio dependentes dessa renda, observou-se (Figuras 2 e 3).

Sendo uma doença crônica, o glaucoma apresenta muitas

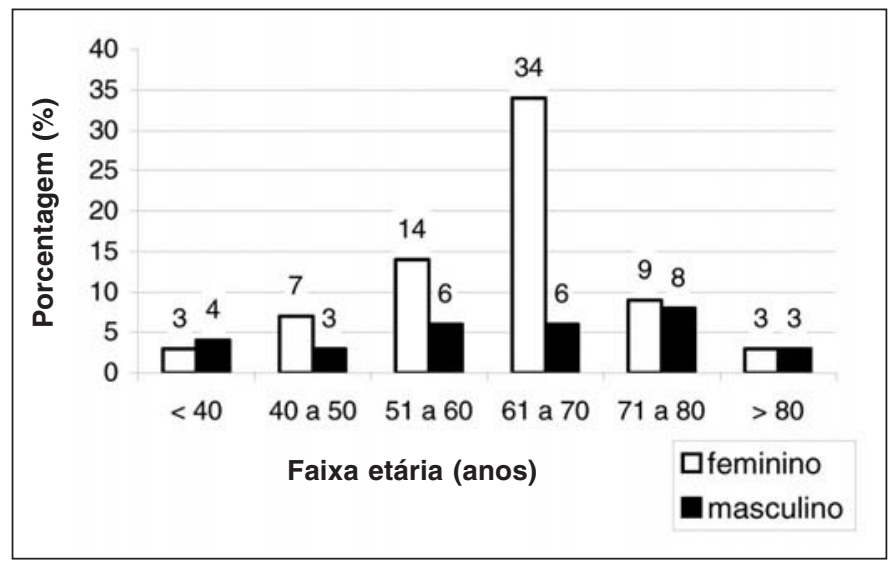

Figura 1 - Relação sexo e idade $(n=100)$ 
co-morbidades. Os pacientes foram questionados em relação à presença de outras patologias, número de medicações em uso nessas co-morbidades e custo mensal com os medicamentos. Observou-se que $78 \%$ dos entrevistados apresentavam co-morbidades (Figuras 4, 5 e 6).

Uma das características importantes na etiologia do glau-

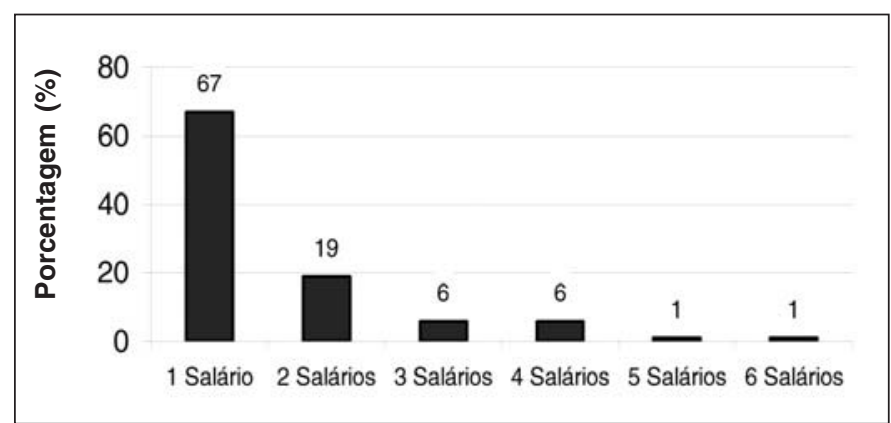

Figura 2 - Renda familiar $(n=100)$

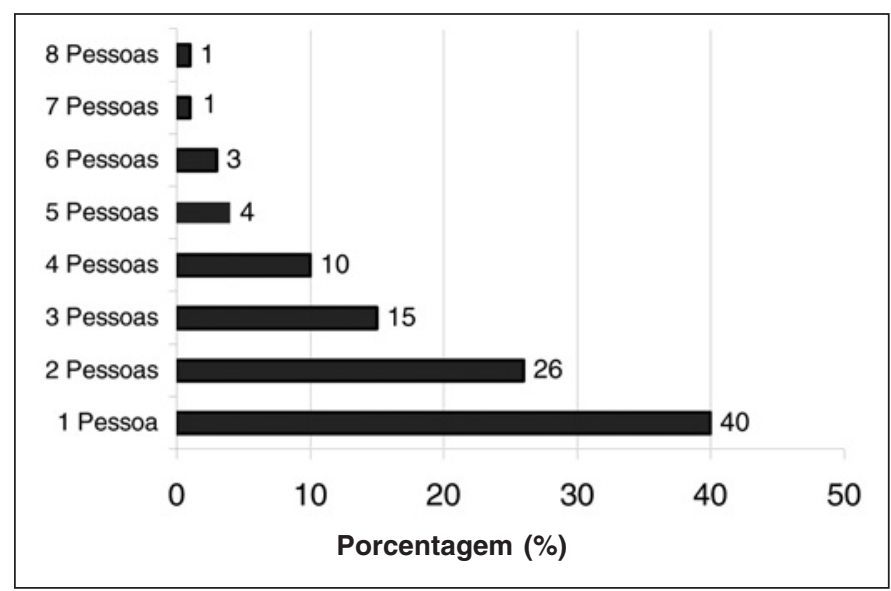

Figura 3 - Número de pessoas no domicílio dependentes da renda familiar $(n=100)$

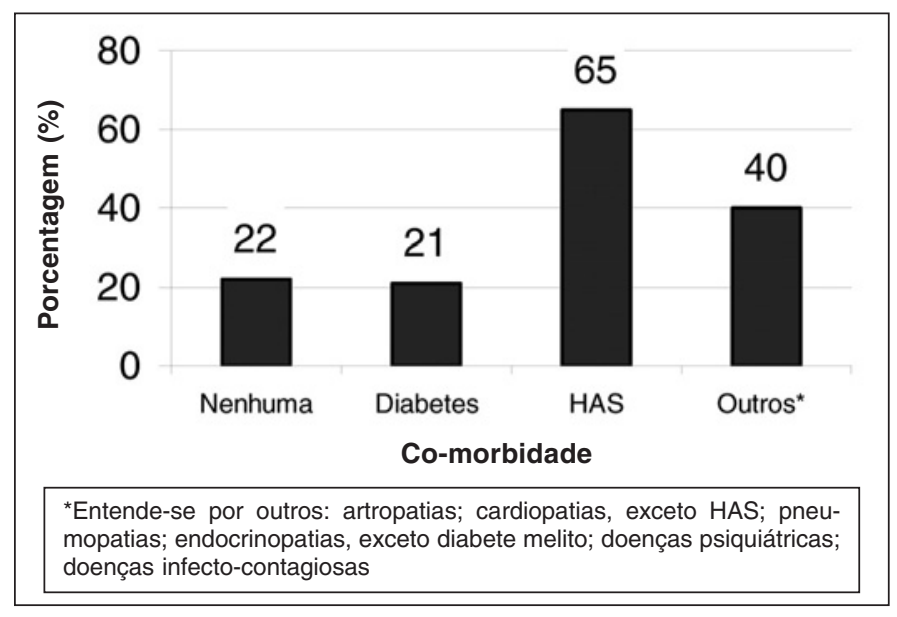

Figura 4 - Co-morbidades associadas ao glaucoma (Foram aceitas mais de uma resposta para o mesmo paciente) coma é a sua história familiar. Observou-se que 30\% dos entrevistados apresentavam historia familiar positiva.

Com relação ao tratamento específico do glaucoma, os entrevistados foram questionados quanto aos tipos farmacológicos prescritos, número de colírios em uso e custo mensal das medicações. Quarenta e um por cento dos entrevistados suspenderam o uso da medicação antiglaucomatosa nos últimos 12 meses devido ao custo (Figuras 7, 8 e 9).

\section{DISCUSSÃO}

O glaucoma é uma neuropatia óptica de causa multifatorial, caracterizada pela lesão progressiva do nervo óptico, culminando em cegueira bilateral em cerca de 8 milhões de indivíduos ${ }^{(1,9)}$. Trata-se de uma doença assintomática que requer investigação especializada pormenorizada. Estima-se que cerca da metade dos casos de glaucoma permanece não diagnosticada ${ }^{(2,10)}$.

Em nosso levantamento de 100 pacientes portadores de glaucoma primário, observou-se uma prevalência maior de glaucoma em indivíduos do sexo feminino (70\%) com média de idade de 61,3 anos. Em uma amostra de 329 pacientes com suspeita de glaucoma do serviço de Oftalmologia da UNICAMP, a prevalência feminina foi de $55 \%$ e a média de idade

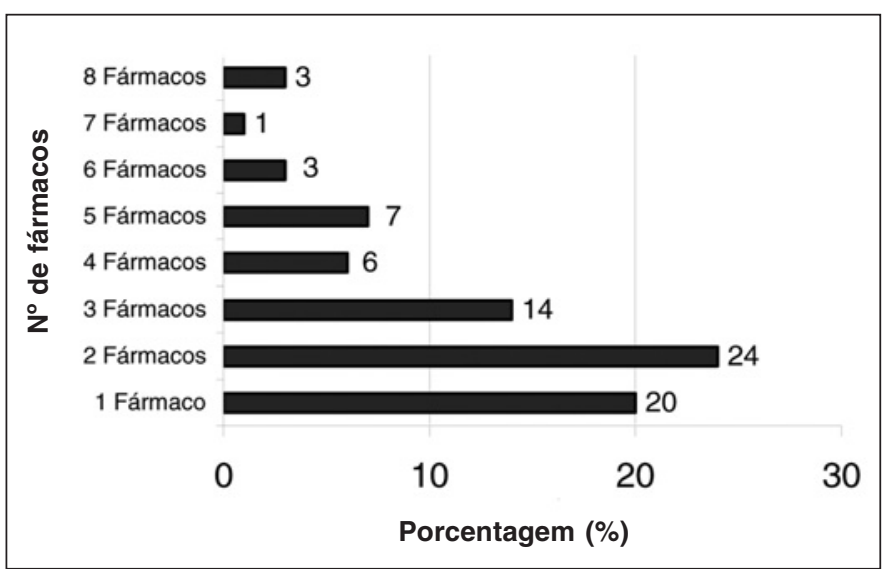

Figura 5 - Número de medicações no tratamento das co-morbidades ( $n=78$ )

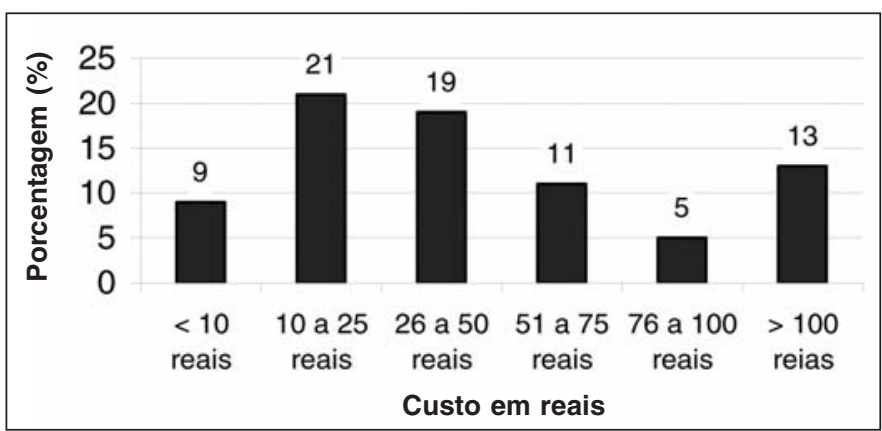

Figura 6 - Custo com as medicações nas co-morbidades $(n=78)$ 


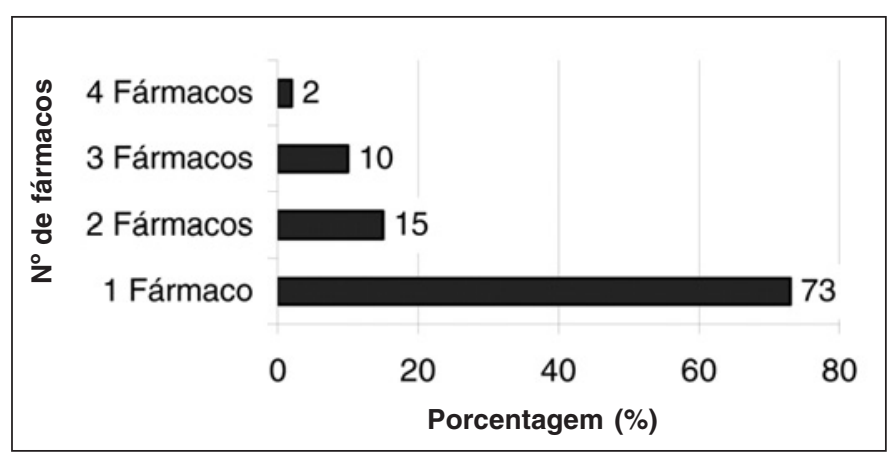

Figura 7 - Número de medicações no tratamento do glaucoma $(n=100)$

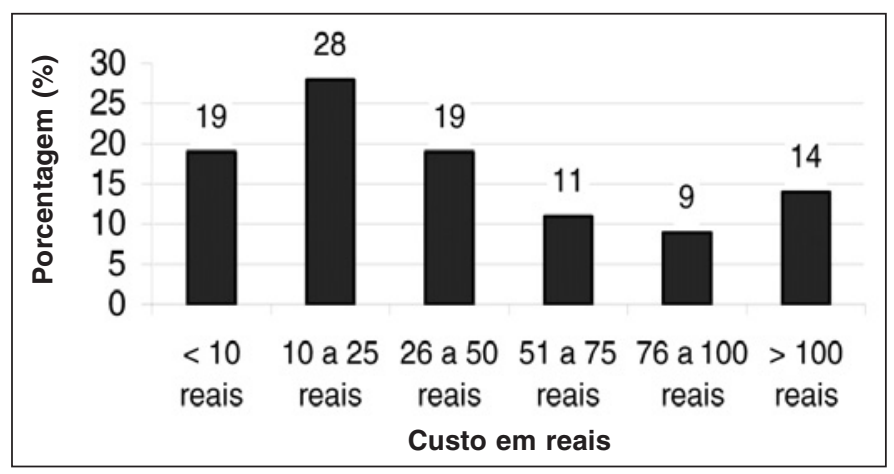

Figura 8 - Custo com as medicações no tratamento do glaucoma $(n=100)$

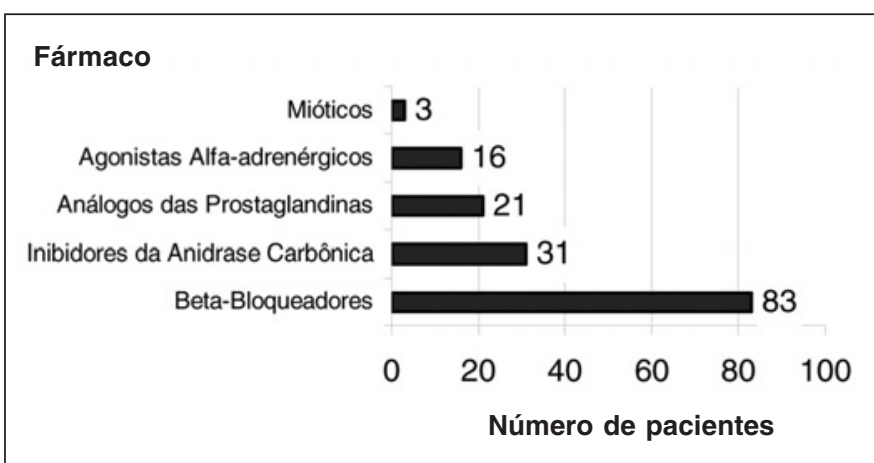

Figura 9 - Tipos farmacológicos em uso no tratamento do glaucoma (As associações farmacológicas foram discriminadas. $O$ paciente pode estar fazendo uso de mais de 1 medicação antiglaucomatosa.)

foi de $60,92 \operatorname{anos}^{(11)}$. Em um estudo retrospectivo de 882 pacientes do setor de Glaucoma da UNIFESP, a idade média foi de 61,7 anos, com cerca de $90 \%$ dos pacientes nas faixas etárias de 40 a 59 anos e acima de 60 anos. O sexo feminino esteve representado em $53,64 \%$ dos casos no mesmo estudo, o que se aproxima com a representatividade feminina de 58\% encontrada em estudo multicêntrico envolvendo 151 pacientes, com média de idade de 66,3 anos $^{(12)}$.

Como toda doença crônica, o glaucoma apresenta problemas de aderência ao tratamento, que é influenciado por vários fatores, dentre eles o custo dos medicamentos, principalmente em populações de baixa renda ${ }^{(9)}$.
É pertinente justificar que o tratamento do glaucoma se mostra associado a um custo elevado em relação à renda familiar da população que freqüente um serviço público ${ }^{(10,13-14)}$. Em nossa população, 84\% dos pacientes eram aposentados e $16 \%$ mantinham algum vínculo profissional. Todos os pacientes avaliados não recebiam benefícios ou doações das medicações. Sessenta e sete por cento de nossa amostra apresentou renda de apenas um salário mínimo e $60 \%$ dos pacientes necessitavam de compartilhar sua renda com os demais membros de seu domicílio. Em um estudo epidemiológico de 146 casos, em 57,5\% deles, apenas o paciente trabalhava, enquanto que a renda era completada por uma pessoa em $62 \%$ dos casos, por duas pessoas em $6,16 \%$ e por 4 ou 5 pessoas em $0,68 \% \%^{(15)}$.

Em nossa amostra, 28\% dos pacientes gastaram de 10 a 25 reais/mês e $29 \%$ gastaram de 26 a 75 reais/mês com o tratamento do glaucoma, recorrendo ao uso de mais de 1 fármaco para o controle adequado da PIO em $27 \%$ dos casos. Um outro estudo apontou que o colírio de maior custo representa um impacto econômico mensal de 29,1\% sobre o valor do salário mínimo. A terapia combinada em apresentações separadas apresenta menor custo que as associações de drogas em apresentação única ${ }^{(16)}$.

É de se esperar maior risco de baixa fidelidade e aderência medicamentosa desses pacientes que destinam parte significante de sua renda ao tratamento. A impossibilidade de aquisição do medicamento resulta no avanço do glaucoma, deteriorando irreversivelmente a visão dos pacientes. Devido à baixa renda familiar e ao alto custo do tratamento, $41 \%$ dos pacientes entrevistados já haviam suspendido o uso dos colírios por falta de condições financeiras para a aquisição dos mesmos, nos últimos 12 meses em nossa amostra. O mesmo foi observado com $45,2 \%$ da amostra de outro estudo ${ }^{(15)}$. A falta de dinheiro foi a principal causa de interrupção do tratamento, ocorrendo em $47,6 \%$ de um outro estudo, situando-se à frente de outros como os efeitos colaterais, ausência de melhora de função visual e presença de co-morbidades ${ }^{(17)}$.

Outro fator que prejudica de maneira importante a adesão dos pacientes ao tratamento antiglaucomatoso é a presença de co-morbidades e o conseqüente gasto mensal com a aquisição de outras modalidades terapêuticas. Em $78 \%$ dos pacientes entrevistados, co-morbidades estiveram presentes, em especial a cardiovascular, representada pela HAS, observada em $65 \%$ dos casos. Cerca de $29 \%$ dos pacientes gastaram acima de 50 reais/mês com o tratamento das co-morbidades, excluindo o custo do tratamento ocular. Vinte por cento da amostra analisada necessitava de adquirir acima de 4 fármacos para o tratamento das co-morbidades. Entretanto, a maioria dos fármacos utilizados nas co-morbidades era adquirida pelo sistema único de saúde.

Em nosso levantamento, 30\% dos pacientes em estudo apresentaram história familiar de glaucoma, o que nos permite inferir que os dependentes da renda dos pacientes em estudo também necessitavam de medicação antiglaucomatosa, o que aumentava substancialmente o custo mensal familiar. Alguns autores encontraram história familiar positiva para glaucoma 
em apenas cerca de $14 \%$ dos pacientes de sua amostra, o que vai de encontro com nosso estudo ${ }^{(11)}$.

O custo médio da medicação hipotensora ocular variou muito ao longo de trinta anos, estando este consideravelmente maior na última década e determinando o predomínio de colírios beta-bloqueadores ${ }^{(18)}$. Nosso estudo confirmou esse dado, já que 53,8\% dos fármacos utilizados no controle do glaucoma eram beta-bloqueadores. Setenta e três por cento dos nossos pacientes fizerem uso de colírio ou droga de administração oral em monoterapia. Em uso de 3 ou 4 medicações, foram encontrados apenas $12 \%$ da população. Esses dados são contrários aos achados do estudo da UNIFESP em que apenas $29 \%$ dos pacientes utilizavam monoterapia no primeiro atendimento de um serviço de glaucoma.

É sabido que a falta de aderência e/ou a baixa fidelidade ao tratamento é um dos maiores fatores de risco para a progressão da doença, o que, em última análise, pode resultar em definitiva e acentuada redução da função visual, com conseqüências sociais graves. $\mathrm{O}$ conhecimento dos pacientes em relação ao glaucoma é relacionado às explicações recebidas e ao nível de escolaridade ${ }^{(15)}$. Isso nos permite inferir que a falta de aderência ao tratamento tem várias vertentes com inúmeras implicações sociais.

\section{CONCLUSÃO}

Pela análise do perfil socioeconômico dos pacientes do presente estudo, ressalta-se a necessidade urgente de adoção de medidas que facilitem o acesso à medicação antiglaucomatosa pelos portadores dessa importante patologia visual. Cabem aos gestores do sistema público de saúde gerenciar os maiores gastos com a reabilitação, procedimentos cirúrgicos e/ou previdência social dos pacientes seqüelados pelo glaucoma. Urge maior educação e esclarecimentos da população com baixo nível socioeconômico.

\section{ABSTRACT}

Purpose: To assess the socioeconomic profile of individuals presenting with primary glaucoma at the ophthalmology service of the university hospital of the Feredal University of Juiz de Fora (MG), Brazil. Methods: Submission of a questionnaire to 100 individuals during consultation in the ophthalmology service of the university hospital of Federal University of Juiz de Fora (MG), Brazil. Results: Eighty-four per cent of individuals were pensioners or retired, $86 \%$ had a monthly income of 1 to 2 salaries, $29 \%$ had been spending 26 to 75 reais per month with glaucoma therapy, $78 \%$ presented with comorbidities and $41 \%$ had already abandoned treatment due to financial difficulties in buying the medication. Conclusion: The low-socioeconomic-level studied population presents with therapeutic compliance difficulties mainly due to financial situation and presence of comorbidities. The authors reinforce the urgent need of adopting sociopolitical measures that facilitate access to antiglaucoma medication, which prompts a better therapeutic compliance.

Keywords: Glaucoma; Glaucoma, open-angle; Ocular hypertension; Glaucoma, angle-closure/epidemiology

\section{REFERÊNCIAS}

1. Coleman AL. Glaucoma. Lancet. 1999;354(9192):1803-10.

2. Krieglstein GK. [Blindness caused by glaucoma]. Ophthalmologe. 1993;90:5546. German.

3. Sperandio AMG. Promoção da saúde ocular e prevenção precoce de problemas visuais nos serviços de saúde pública. Rev Saúde Pública. 1999;33(5):513-20.

4. Walckiers D, Sartor F. Results of an epidemiological study on drug-treated intraocular hypertension in Belgium. J Clin Epidemiol. 1996;49(4):489-93.

5. Amaral Filho JM, Moreira RA, Silva LM, Vasconcelos JP, Rocha EM, Costa VP, et al. Custo mensal de medicações antiglaucomatosas no Brasil. Arq Bras Oftalmol. 1999;62(2):123-6.

6. Prado FC, Ramos JA, Valle JR. Atualização Terapêutica. São Paulo: Artes Médicas; 2003.

7. Associação Brasileira dos Portadores de Glaucoma (ABRAG). [sítio na Internet]. São Paulo: ABRAG. [citado 2006 Nov 11]. Disponível em: http://www. abrag.com.br/

8. Hoevenaars JG, Schouten JS, Van den Borne B, Beckers HJ, Weberd CA Socioeconomic differences in glaucoma patients knowledge, need for information and expectations of treatments. Acta Ophthal Scand. 2006;84(1):84-91.

9. Wilson MR, Martone JF. Epidemilogy of chronic open angle glaucoma. In: Ritch R, Shields MB, Krupin T. The glaucomas. $3^{\text {rd }}$ ed. St Louis: Mosby; 1996. p.407-45

10. Urbano AP, Freitas TG, Arcieri ES, Urbano AP, Costa VP. Avaliação dos tipos de glaucoma no serviço de oftalmologia da UNICAMP. Arq Bras Oftalmol. 2003;66(1):61-5.

11. Oliveira A, Paranhos Júnior A, Prata Júnior J. Características dos pacientes atendidos pela primeira vez no Setor de Glaucoma da Universidade Federal de São Paulo - UNIFESP. Arq Bras Oftalmol. 2003;66(6):785-90.

12. Lee PP, Walt JG, Doyle JJ, Kotak SV, Evans SJ, Budenz, DL, et al. A multicenter, retrospective pilot study of resource use and costs associated with severity of disease in glaucoma. Arch Ophtalmol. 2006;124(1):12-9

13. Bartosova L, Kraus H, Ruzickova E. [Working ability of patients with various forms and stages of glaucoma]. Cesk Oftalmol. 1989;45(2):127-37. Czech.

14. Pedroso L, Carvalho Jr. ES, Paranhos Junior A, Prata Jr JA, Mello PA. Custo real do tratamento do glaucoma para o paciente. Arq Bras Oftalmol. 1999;62 (6):677-82.

15. Silva LM, Vasconcelos JP, Temporini ER, Costa VP, Kara-José N. Tratamento clínico do glaucoma em um hospital universitário: custo mensal e impacto na renda familiar. Arq Bras Oftalmol. 2002;65(3):299-303.

16. Stillitano IG, de Lima MG, Ribeiro MP, Cabral J, Brandt CT. Impacto econômico do custo de colírios no tratamento do glaucoma. Arq Bras Oftalmol. 2005;68(1):79-84.

17. Costa VP, Vasconcellos JP, Pelegrino M, Kara-José N. O que os pacientes sabem sobre glaucoma? Arq Bras Oftalmol. 1995;58(1):36-41.

18. Souza Filho JP, Dias AB, Lima Filho AA, Sartori MP, Martins MC. A evolução do mercado farmacêutico brasileiro no tratamento do glaucoma nos últimos 30 anos. Arq Bras Oftalmol. 2003;66(6):811-7. 